\title{
Migration und Entwicklung : eine Reflexion der Fédération genevoise de coopération
}

Yanik Marguerat et Christelle Genoud

\section{(2) OpenEdition}

Édition électronique

URL : http://journals.openedition.org/sjep/356

DOI : $10.4000 /$ sjep.356

ISSN : 1663-9677

Éditeur

Institut de hautes études internationales et du développement

Édition imprimée

Date de publication : 1 décembre 2008

Pagination : 249-255

ISBN : 978-2-940415-08-3

ISSN : 1660-5926

Référence électronique

Yanik Marguerat et Christelle Genoud, « Migration und Entwicklung : eine Reflexion der Fédération genevoise de coopération », Schweizerisches Jahrbuch für Entwicklungspolitik [En ligne], 27-2 | 2008, mis en ligne le 31 mai 2010, consulté le 08 septembre 2020. URL : http://journals.openedition.org/sjep/ 356 ; DOI : https://doi.org/10.4000/sjep.356 


\title{
Migration und Entwicklung: eine Reflexion der Fédération genevoise de coopération
}

\author{
Yanik Marguerat und Christelle Genoud*
}

n den letzten Jahren haben sich zahlreiche internationale Instanzen zur komplexen Wechselwirkung zwischen Migration und Entwicklung Gedanken gemacht und an diesem Thema gearbeitet. Hierzu lassen sich insbesondere der von den Vereinten Nationen im Jahr 2005 eingesetzte hochrangige Dialog über Migration und Entwicklung (UN High Level Dialogue on Migration and Development) sowie die Zusammenkünfte des Weltforums für Migration und Entwicklung (Global Forum on Migration and Development) 2007 in Brüssel und 2008 in Manila erwähnen. Einige grosse Schweizer Nichtregierungsorganisationen haben ebenfalls einen Reflexionsprozess zu dieser Frage eingeleitet (vor allem das Schweizerische Rote Kreuz und Caritas ${ }^{1}$ ), jedoch hat die Zivilgesellschaft in der Schweiz im Grossen und Ganzen diesen Forschungsbereich bislang noch wenig erkundet. Die gesamte Betrachtung muss ferner im Licht der politischen Lage in der Schweiz betrachtet werden - wo die Migrantenverbände, deren Mitglieder und die Zivilgesellschaft im Allgemeinen derzeit in einem gegenüber Flüchtlingen und aussereuropäischen Ausländern verschärften und restriktiveren Gesetzesrahmen vorgehen.

In diesem Kontext beschloss die Fédération genevoise de coopération (FGC), eine Genfer Dachorganisation, der 48 im Bereich der Entwicklungszusammenarbeit und der Sensibilisierung tätige Nichtregierungsorganisationen angehören, mit ihren Mitgliedern an der Thematik „Migration und Entwicklung“ zu arbeiten $^{2}$. Hierzu organisierte die FGC einerseits eine dreitägige Veranstaltung, das zweite Genfer Solidaritätsforum (2e Carrefour genevois de la solidarité), die sich mit diesem Thema befasste. Die Fédération genevoise de coopération wollte die Stimmen des Südens in den Vordergrund stellen. Dies tat sie, indem sie mehrere Migranten aus den Ländern des Südens, welche die Migration als Realität täglich erleben, - namentlich verschiedene Partner aus Westafrika - zur Meinungsäusserung einlud. Parallel dazu wurde eine qualitative Studie über die

* Yanik Marguerat, Kommunikationsbeauftragter, Fédération genevoise de coopération (FGC).

Christelle Genoud, Ökonomin, im Auftrag der Fédération genevoise de coopération (FGC).

1 Bettina Zeugin, Où en est la politique migratoire de la Suisse?, Stellungnahme, Studiensektor, Luzern, Caritas Schweiz, Mai 2007, <http://www.caritas.ch>; Jean-François Giovannini (Ratsmitglied des Roten Kreuzes), Einführungsansprache zur Jahreskonferenz des Schweizerischen Roten Kreuzes mit dem Titel: „La migration, une contribution au développement“, 30. November 2006, $<$ http:// www.crg.org>. Aus dieser Konferenz ging die Publikation Schweizerisches Rotes Kreuz (Hrsg.), Migration, ein Beitrag zur Entwicklung ?, Zürich, Seismo, 2007, hervor.

2 Die FGC und ihre Mitgliedsverbände arbeiten mit Partner-NRO im Süden zusammen, um Ungleichheiten zu bekämpfen und Gesellschaften und Völkern zu mehr Wahlfreiheit bei der Schaffung besserer wirtschaftlicher, sozialer und kultureller Lebensbedingungen zu verhelfen. Die Verbände sind sehr unterschiedlich in Bezug auf ihre Ziele, Strukturen und Tätigkeiten, jedoch teilen sie alle die Vision einer globalen nachhaltigen Entwicklung, die auf den Werten von sozialer Gerechtigkeit, Chancengleichheit und menschlicher Würde beruht. Für weitere Informationen siehe : <http://www.fgc.ch>. 
Beziehungen zwischen Migration und Entwicklungshilfeprojekten der FGCMitgliedsverbände durchgeführt. Dieses Vorgehen erfolgt im Rahmen einer Auswertung der Erfahrungen, bei der eine Etappe darin bestand, ein internes Diskussionsforum unter den Mitgliedern der FGC zu organisieren, welche die Ergebnisse der Studie erörterten. Eine weitere Etappe wird die Herausgabe des Berichts des Forums Carrefour genevois de la solidarité sein, der auf der Website der FGC zugänglich gemacht wird ${ }^{3}$. All diese Elemente werden mit dem Kontext in Verbindung gebracht und ergänzen den zentralen Teil des Vorgehens, d.h. die Studie über die von der FGC geförderten Projekte, von der ein Teil der Ergebnisse nachstehend präsentiert wird ${ }^{4}$.

\section{Einige Ergebnisse der Studie „Migration und Entwicklungshilfeprojekte“}

Ein erstes wichtiges - indirektes - Resultat der Studie besteht darin, eine Sensibilisierung für die Migrationsthematik bei den hier in Genf befragten Projektverantwortlichen und ihren Partnern im Süden bewirkt zu haben. Die genannten Akteure konnten sich beim Ausfüllen der im Rahmen der Studie verteilten Fragebögen über das Interesse informieren, das derzeit beispielsweise den Geldüberweisungen der Migranten in die Entwicklungsländer oder den Aktionen der Diaspora entgegengebracht wird. Andere lernten durch die Studie das Migrationskonzept kennen, so wie es von der Internationalen Organisation für Migration (IOM) definiert wird. Dieses Konzept umfasst nicht nur die freiwilligen Wanderungen von Arbeitnehmern, sondern auch die „erzwungenen“ Wanderungen und betrifft somit auch Flüchtlinge, Vertriebene und Opfer von Menschenschmuggel. Durch die Entscheidung, auch das Phänomen der Landflucht und die mit der Trockenzeit einhergehenden temporären Wanderungen in die Reflexion einzubeziehen, konnten Zusammenhänge zu vielen geförderten Projekten hergestellt werden, die von den Verantwortlichen vielleicht nicht von Anfang an erkannt worden waren.

Ein weiteres interessantes Resultat, das sich aus der Untersuchung ergab, betrifft die Aneignung des Themas „Migration“ durch die Verbände der FGC und ihre Mitglieder. Man kann sich in der Tat fragen, warum sich die Leiter der Entwicklungsprojekte die Migrationsthematik nicht besser zu eigen gemacht haben. Eine der Erklärungen liegt vielleicht in dem Medienrummel, der im Zusammenhang mit den Phänomenen der Nord-Süd-Migration gemacht wird. Hinter den dramatischen Bildern von den spanischen Exklaven Ceuta und Melilla oder den Ertrunkenen jener Boote, welche die Küsten Europas zu erreichen versuchen, verbirgt sich eine andere, viel weniger sichtbare, aber genau so belastende Realität, nämlich dass rund 80 Prozent der Flüchtlinge ${ }^{5}$ und über 60 Prozent der Migranten in den Entwicklungsländern ${ }^{6}$ leben.

$3<$ http://www.fgc.ch/carrefours.php>.

4 Die Studie basiert zum einen auf qualitativen Gesprächen mit Projektverantwortlichen in der Schweiz und mit Partnern vor Ort, zum anderen auf den Angaben aus 30 Fragebögen, die von den Mitgliedsverbänden der FGC ausgefüllt zurückgesandt wurden.

5 Die Zahl der Flüchtlinge ist weltweit von 18,5 Millionen im Jahr 1990 auf 13,5 Millionen im Jahr 2005 zurückgegangen. 10,8 Millionen Flüchtlinge halten sich in den Entwicklungsländern auf, davon 7,8 Millionen in Asien und 3 Millionen in Afrika. (Assemblée générale des Nations Unies, Migrations Internationales et Développement, Rapport du Secrétaire général, A/60/871, Mai 2006, S. 46).

6 Catherine Wihtol de Wenden, Atlas des migrations dans le monde, Paris, Autrement, 2005. 
Die internationale Migration ist eine komplexe Erscheinung, die eine Vielzahl von Faktoren umfasst und zahlreiche Auswirkungen auf unsere Welt hat. Wenn die Migration in ihrer vollumfänglichen Definition betrachtet und gut erläutert wird, sind die Projektverantwortlichen, die mit den Ländern des Südens arbeiten, besser imstande, die Migration zu konzeptualisieren, sie zu verstehen und bei ihren Aktionen zu berücksichtigen, wie sich aus der Studie der FGC ergab.

Die Aneignung dieses Konzepts könnte letztlich das Mittel sein, um effizientere, lebensfähige Projekte umzusetzen, die einer gegebenen sozioökonomischen Situation angepasst wären, da sie den komplexen Migrationsfaktor berücksichtigen würden.

Obwohl die Studie eine gewisse mangelnde Aneignung der Migrationsthematik ans Licht brachte, enthalten einige der untersuchten Projekte, welche die Dimension der Migration in der Entwicklung gut integriert haben, sehr relevante Informationen.

Das erste Beispiel betrifft ein vom Verband „Les Jardins de Cocagne - Solidarité Nord et Sud" durchgeführtes Projekt, das zum Ziel hat, die Entwicklungsinitiativen und die Stärkung der Zivilgesellschaft in der Gemeinde Kothiary im Senegal zu fördern ${ }^{7}$. Die Gemeinde zählt eine grosse Anzahl von Emigranten, die sich vor allem in Frankreich niedergelassen haben, und hat die Zusammenarbeit mit Migranten in ihren lokalen Entwicklungsplan integriert. Demzufolge treffen sich Vertreter der lokalen Behörden und der Zivilgesellschaft sowie Mitarbeiter der Organisation „Les Jardins de Cocagne“ und Mitglieder des Verbands der Einwohner von Kothiary in Frankreich hier im Norden oder vor Ort zum gemeinsamen Vorgehen. Die Beteiligten sind sich überdies der Bedeutung bewusst geworden, Kommunikationsmittel mit einem regelmässigen Informationsaustausch zwischen dem Norden und dem Süden einzusetzen. Ein konkretes Beispiel dieser Zusammenarbeit ist die Überweisung von Geldern der in Frankreich etablierten Emigranten an die lokalen Spar- und Kreditkassen, die dank der Unterstützung des Verbands geschaffen wurden. Das Projekt ermöglicht es zudem, Gemeinschaftsläden zu gründen, um gegen die zu hohen Preise der Händler anzugehen, Saatgutbanken einzurichten und in die Bereiche Wasser, Bildung und Gesundheitswesen zu investieren. Diese neuen Praktiken stellen einen Bruch mit der Vergangenheit dar, da die von den Emigranten überwiesenen Gelder zuvor zwar zum Bau von Strassen und Gesundheitszentren, aber auch für unproduktive Ausgaben (Häuser, Viehzucht zu Prestigezwecken) eingesetzt wurden.

Das zweite Beispiel betrifft das mit Einwohnern des Gebietes der Grossen afrikanischen Seen durchgeführte Experiment, das sich in der „Genfer Initiative für den Frieden in der Region der Grossen Seen“ niederschlug ${ }^{8}$. Diese Initiative nahm die Form eines Netzwerks von führenden Persönlichkeiten aus Burundi, aus dem Nord- und Süd-Kivu und Ruanda an. Ein im Jahr 2003 veranstaltetes Seminar, dessen Ziel es war, konkrete Handlungswege zur Friedensförderung zu erarbeiten, stand am Anfang dieses Netzwerks. Das Hauptanliegen des Seminarveranstalters war, dass die Mitglieder der Diaspora selber die Spannungen

7 Siehe auch das Gespräch mit Ibrahima Thioye, einem mauretanischen Partner der Organisation „Les Jardins de Cocagne“", in diesem Dossier.

8 Das Projekt wurde von der Dritte-Welt-Kommission der Katholischen Kirche und Eirene Schweiz durchgeführt. 
abbauen, statt sie anzufachen. Nach seiner Ansicht war es unerlässlich, Massnahmen bei den Migranten dieses Gebiets und mit ihnen zusammen einzuleiten. Vor allem war der langwierige Prozess zur Ermittlung der Seminarteilnehmer beispielhaft: Der Verantwortliche des Projekts, ein in Genf etablierter Ruander, traf sich mit zahlreichen Persönlichkeiten, um die Seminarteilnehmer zu identifizieren und sich von ihrer tatsächlichen Fähigkeit zur Überwindung der Spannungen zu überzeugen. Das Treffen dieser Personen war das Ergebnis zweijähriger Aushandlungen. Ferner war die Qualität des Austauschs zwischen den Teilnehmern bei diesem Experiment wesentlich: Die Mitglieder berichteten über ihre verschiedenen Lebenserfahrungen, bevor gemeinsame Aktionsstrategien ausgearbeitet wurden. Dieser Erfahrungsaustausch hat starke soziale Verbindungen zwischen ihnen geschaffen, die auch noch fünf Jahre nach ihrem ersten Treffen bestehen und zur Umsetzung von Entwicklungsprojekten durch das Netzwerk selbst führten. Hierzu lässt sich beispielsweise die kürzliche Veranstaltung eines Forums nennen, das diesmal die junge Diaspora der Grossen Seen im Senegal zusammenbrachte, oder auch die Verbreitung der Erfahrungsberichte vom Jahr 2003 in Form einer Ausstellung, die in den wichtigsten Städten des Gebiets der Grossen Seen gezeigt wurde ${ }^{9}$.

\section{Mit Migranten arbeiten?}

Die Erfahrungen dieser beiden Nichtregierungsorganisationen mit Migranten sind auch für andere interessant, und es scheint heute wichtig, die Lehren aus einigen Initiativen zu ziehen. Durch derzeit in der Schweiz gesammelte Daten soll diese Art von Zusammenarbeit ebenfalls erleichtert werden. Hierzu lassen sich die Informationen anführen, die betreffend mehrere in der Schweiz bestehende Migrantengemeinschaften und ihre Beteiligung an der Entwicklung ihrer Herkunftsländer zusammengetragen werden ${ }^{10}$, oder die Erstellung von Datenbanken, in denen die in der Schweiz lebenden qualifizierten Migranten aus Entwicklungsländern registriert werden ${ }^{11}$. All diese Instrumente werden es längerfristig ermöglichen, die Verbände im Norden besser auszustatten, die mit Migranten arbeiten wollen, welche über Kenntnisse und relevante Erfahrungen verfügen.

In Genf leben zahlreiche Emigranten: Es sind Arbeitnehmer, politische oder Wirtschaftsflüchtlinge, internationale Beamte und Personen ohne Rechtsstatut. Sie bilden ein Reservoir an Kompetenzen und Fachwissen, das den Entwick-

9 Das Vorgehen führte zur Veröffentlichung eines Buches und zur Veranstaltung einer Ausstellung mit dem Leitmotiv: „Dépasser la haine, construire la paix : un choix, un engagement“ (Hass überwinden und Frieden schaffen: eine Wahl und ein Engagement) zu dieser Thematik. Die Grundlage dafür bildeten die erlebten und geschilderten Erfahrungen von Bewohnern des Gebiets der Grossen Seen, die sich für die Förderung des Friedens und für ein besseres Zusammenleben einsetzen.

10 Das heisst eine Studie des Staatssekretariats für Wirtschaft (SECO) über die Geldüberweisungen der serbischen Diaspora, eine Studie der Eidgenössischen Technischen Hochschule Lausanne (ETHL) über die dominikanische Diaspora in der Schweiz sowie ein Projekt für die Zusammentragung von Daten betreffend die Diaspora aus Afrika südlich der Sahara, das von der Direktion für Entwicklung und Zusammenarbeit (DEZA) geleitet wird.

11 Eine erste Datenbank betreffend die kolumbianischen, indischen und südafrikanischen Emigranten wird derzeit bei der ETH Lausanne eingerichtet (<http://www.cooperation.epfl.ch/ScientificDiasporanetwork>); eine zweite Datenbank afrikanischer Kompetenzen wird vom Verband „Regards Africains" in Genf erstellt. 
lungsorganisationen ein interessantes Potenzial sowohl für das Vereinsleben als auch für die Ausarbeitung von Projekten bieten kann.

Einige Mitgliedsorganisationen der FGC sind der Meinung, dass die Integration der Emigranten in ihren Verband wichtig ist. Im Fall der Genfer Initiative für den Frieden im Gebiet der Grossen afrikanischen Seen wurden der gesamte Definitionsprozess und die Inbetriebnahme des Projekts von Personen aus der Region konzipiert. Hierbei handelt es sich um ein erfolgreiches Beispiel egalitärer Partnerschaft, aber es muss auch akzeptiert werden, dass dieser Partnerschaftsansatz Risiken mit sich bringt. Selbst wenn die Migration im vorliegenden Fall ein Potenzial darzustellen scheint, muss man in der Folge auch in Betracht ziehen, dass die Entscheidungsbefugnisse geteilt werden müssen, dies sowohl was die Finanzen betrifft, als auch die Art, die Projekte zu konzipieren und durchzuführen.

Es ist jedoch interessant zu vermerken, dass in einigen spezifischen Fällen gewisse in Genf tätige Entwicklungsverbände beschlossen haben, die Projektbetreuung nicht Verantwortlichen aus den Ländern anzuvertrauen, in denen die Projekte umgesetzt werden. Die Erfahrung hat sie nämlich gelehrt, dass die Kluft zwischen diesen Projektbeauftragten und den begünstigten Bevölkerungsgruppen so tief war, dass sie die ordnungsgemässe Abwicklung der Aktivitäten beeinträchtigen konnte. Im gleichen Sinne haben andere Verbände festgestellt, dass die Zusammenarbeit mit Migranten aus den Einsatzgebieten der Projekte nicht immer glücklich war und zuweilen grosse Schwierigkeiten aufwarf. Diese Feststellung scheint noch ausgeprägter zu sein, wenn sich das betreffende Land in einem Konflikt befindet. So erklärt ein Verband, der Friedenförderungsprojekte in Kooperation mit der israelischen und der palästinensischen Zivilgesellschaft entwickelt, er ziehe es vor, keine Zusammenarbeit mit den Migrantenverbänden dieser beiden „Länder“ in Genf einzugehen, um die Unparteilichkeit der Projekte zu bewahren. Der betreffende Verband, der sich zunächst die Frage nach dem Nutzen einer solchen Zusammenarbeit gestellt hatte, war durch Erfahrung zum Schluss gekommen, dass sich der Einsatz nicht lohne. Diese Zurückhaltung scheint vor allem mit Konfliktsituationen verbunden zu sein, jedoch kann auch das Bestehen sozioökonomischer Diskrepanzen zwischen der begünstigten Bevölkerung und den in Genf niedergelassenen Emigranten zu unterschiedlichen Interessen führen und sich für die Projekte als kontraproduktiv erweisen.

Hingegen haben andere Mitgliedsverbände der FGC beschlossen, die Betreuung der Aktionen Emigranten der Gebiete anzuvertrauen, denen die Projekte zugute kommen, da sie der Meinung sind, dass die Kenntnis der Verhältnisse vor Ort, die Erfahrung und die Motivation der Betroffenen wirklich von Vorteil seien. Zudem zählen zwei Drittel der Mitgliedsorganisationen der FGC unter ihren Mitgliedern Personen aus den Regionen, in denen die Projekte entwickelt werden.

Eine schwierige politische Wahl

Die von der FGC in ihren Reihen lancierte Reflexion über die Zusammenhänge zwischen Migration und Entwicklung setzt voraus, dass die Organisation selbst früher oder später zu dieser heiklen Frage Stellung nimmt. In der Tat stellt die einfache Tatsache, diese Problematik zum eigenen Anliegen zu machen, einen 
politischen Akt dar. Gegen den vorherrschenden soziopolitischen Kontext angehend zu behaupten, die Migration stelle ein echtes Potenzial dar, ist eine politische Stellungnahme. Somit muss man sich auch die Mittel geben, um zu dieser Position zu stehen.

Im Rahmen des zweiten Solidaritätsforums $\left(2^{e}\right.$ Carrefour genevois de la solidarité) vom Januar 2008 wollte die FGC die Migration unter einem positiven Aspekt präsentieren und zeigen, wie sehr dieses Phänomen einem Austausch von Reichtümern zwischen zwei Welten gleichkommt. Die Migranten kommen in die Schweiz, um einem chronischen Arbeitskräftemangel abzuhelfen; sie stellen eine Abhilfe für das Altern unserer Gesellschaft dar und sind eine Bereicherung für den Norden und den Süden, sowohl in finanzieller als auch in kultureller Hinsicht. Im Einzelnen drehten sich die Diskussionen unter anderem um die Geldüberweisungen der Emigranten und die Unterstützung für gewisse Projekte von Migrantenverbänden, welche eine positive Wirkung auf die Entwicklung der Herkunftsländer haben können.

Die Partner aus dem Süden, die auf dem Solidaritätsforum das Wort ergriffen, vertreten jedoch eine viel kritischere Lesart der internationalen Migration. So gab es zahlreiche afrikanische Stimmen, die auf die Kehrseite der Migrationsmedaille hinwiesen, welche als Folge des Fehlschlags der staatlichen Entwicklungspolitik hingestellt wird. So erklärte die Schriftstellerin und frühere Ministerin für Kultur und Tourismus Malis, Frau Aminata Traoré: „Niemand würde sich ins Meer stürzen, wenn Entwicklung stattgefunden hätte. Bislang hat die Entwicklung darin bestanden, neoliberale Reformen durchzusetzen. Das gesamte Problem ergibt sich aus der Tatsache, dass man dieses Gesellschaftsmodell mit der Hilfe von Führungskräften durchsetzen will, die beschlossen haben, Musterschüler des Internationalen Währungsfonds und der Weltbank zu sein. Es hat keinerlei Entwicklung in Afrika gegeben. Es hat nur Profit gegeben. Unsere Existenz wird von Marktgesetzen geleitet, die uns niemals günstig waren."12

In diesem Kontext müssen sich die Zivilgesellschaft und die Nichtregierungsorganisationen positionieren und im Zusammenhang mit der Frage der Migration und der Entwicklung ihre Wahl treffen. Für die Entwicklungsorganisationen des Nordens ist jegliche politische Stellungnahme in dieser Debatte sehr heikel. Einerseits muss darauf geachtet werden, nicht den Diskurs einer gewissen populistischen Rechten zu legitimieren, welche die Entwicklungszusammenarbeit unterstützen würde, wenn diese es wirklich erlauben würde, die Migration zu bremsen. Andererseits muss es gelingen, die Migration in ihrem positivem Licht darzustellen, ohne jedoch die Tatsache zu übergehen, dass die „Migration der Verzweiflung" alljährlich Tausende von Ertrunkenen an die Strände Europas spült und die Eltern-Kind-Beziehungen jener Migranten und Migrantinnen fragilisiert, die ihre Kinder im Herkunftsland zurücklassen um - just zum Wohl ihrer Familie - zur Arbeitssuche nach Europa zu kommen ${ }^{13}$. Auch ist interessant, auf die jüngsten Studien hinzuweisen, die das Phänomen des Migration

12 Unsere Übersetzung (AdR). Siehe auch das neueste Buch von Aminata Traoré: L'Afrique humiliée, Paris, Fayard, 2008.

13 Eine Vertreterin der Gruppe zur Unterstützung der Papierlosen in Genf hat sich an einem Workshop anlässlich des zweiten Solidaritätsforums gefragt, ob ihre Anstrengung der Mühe wert sei : „Mit seinen Kindern kann man diese verlorenen Jahre nie wieder aufholen.“ 
Hump herausgestellt haben, das zeigt, dass die Entwicklung die Migration in einer ersten Phase fördern kann.

Im Rahmen ihrer Ausführungen auf dem zweiten Solidaritätsforum hat Aminata Traoré den Begriff „Migration“ als solchen infrage getellt: Warum werden die 600000 Schweizer in Ausland nicht - wie alle ausländischen Staatsangehörigen im Norden - als „Migranten“ bezeichnet? Dies zeigt nach Ansicht von Frau Traoré deutlich die diskriminierende Verwendung des Begriffs „Migrant“ durch die Länder des Nordens.

Einige meinen, dass viele Akteure in der Schweiz heute aus Opportunismus über die Migrationsthematik diskutieren. In einem politischen Kontext, der gegenüber der Entwicklungshilfe sehr kritisch eingestellt ist, hat die Hilfe Tendenz, sich auf eine zweckmässige Vision zu beschränken: als Instrument, um die Lebensbedingungen der benachteiligten Bevölkerungsgruppen zu verbessern, die dadurch nicht zur Auswanderung nach Europa verleitet würden. Die Hilfwerke müssen darüber wachen, dass die schweizerische Entwicklungszusammenarbeit nicht instrumentalisiert wird, und dass sie nicht nur zu einem Werkzeug wird, um die Interessen der Schweiz zu fördern. Die Situation ist seit der Verschärfung des Asyl- und des Ausländergesetzes noch komplexer geworden, und diese innenpolitische Frage muss von den Migrantenverbänden und den für die Interessen der Migranten eintretenden Vereinigungen angegangen werden. Die Fédération genevoise de coopération und die Organisationen für internationale Solidarität müssen die Debatte auch auf ihrer Ebene aufgreifen, indem sie die mit der Migration verbundenen Chancen präsentieren, ohne sie zu idealisieren, und indem sie die Möglichkeiten fördern, diese posiviten Effekte auf die Gesellschaften der Länder des Südens zu maximieren. Bezüglich dieser äusserst komplexen und sensiblen Problematik muss sich die Zivilgesellschaft aktiv einsetzen, konkrete Lösungen vorschlagen und gleichzeitig weiterhin zur Reflexion und zum Gedankenaustausch in diesem Bereich beitragen.

Letztendlich werden die Ergebnisse im Entwicklungsbereich wohl an der Wahl der betroffenen Bevölkerung gemessen werden, d.h. an der freien Entscheidung, auszuwandern oder nicht, ohne durch Armut oder aus Verzweiflung auf der Suche nach einer sehr fraglichen besseren Zukunft in ferne Länder getrieben zu werden. 